\title{
Comparative Study of Auto Plan and Manual Plan for Nasopharyngeal Carcinoma Intensity-Modulated Radiation Therapy
}

This article was published in the following Dove Press journal: Cancer Management and Research

\author{
Xin Xin (D) \\ Chuandong Cheng \\ Churong $\mathrm{Li}$ \\ Jie Li \\ Pei Wang \\ Gang Yin \\ Jinyi Lang $(\mathbb{C}$
}

Department of Radiation Therapy, Sichuan Cancer Hospital, Radiation Oncology Key Laboratory of Sichuan Province, Chengdu, People's Republic of China
Correspondence: Gang Yin; Jinyi Lang Department of Radiation Therapy, Sichuan Cancer Hospital, Radiation Oncology Key Laboratory of Sichuan Province, No. 55, The 4th Section of Renmin South Road, Chengdu, People's Republic of China

Email cxqyguestc@I63.com; langjy610@163.com
Purpose and Objective: Auto planning might reduce the manual time required for the optimization and could also potentially improve the overall plan quality. The aim of this study is to demonstrate the statistical comparison of automatic (AU) and manually (MA) generated nasopharyngeal carcinoma (NPC) intensity-modulated radiation therapy (IMRT) plans.

Materials and Methods: The study included 105 nasopharyngeal carcinoma patients, admitted to our hospital. The patients underwent IMRT treatments. The clinically delivered plans were performed with Eclipse (Version 11.0) using manual optimization. The same plans were optimized successively in Pinnacle ${ }^{\mathrm{TM} 3}$ (version 9.10) treatment planning system using the auto plan software package module. D95 (dose of 95\% volume) and D98 (dose of 98\% volume) were calculated for the targets and maximum dose (Dmax) and mean dose (Dmean) for the organ at risks (OARs); moreover, the average doses of each target and OARs for 105 patients were evaluated.

Results: There is no significant difference in the homogeneity of the target between AU and MA treatment plans, while a significant difference is observed for what is concerning the OARs or most of OARs in 105 patients, OAR doses were significantly reduced in AU plan. For OARs which have no significant difference between AU and MA plans are highlighted, the mean dose of OARs in AU plans was at least not higher than MA plans.

Conclusion: Nasopharyngeal carcinoma IMRT plans made by an automatic planning tool met the clinical requirements for target prescription dose; moreover, the dose of normal tissues was lower than in MA plans. Clinical physicists' time can be saved and the influence of factors such as the lack of experience in treatment planning can be avoided.

Keywords: auto planning, manual planning, nasopharyngeal carcinoma

\section{Introduction}

Head and neck cancer is one of the most common malignant tumors all over the world. Because of the high sensitivity to radiotherapy and chemotherapy, radiotherapy with concurrent chemotherapy has become the main method of treatment of nasopharyngeal carcinoma, and especially with the introduction of IMRT, ${ }^{1}$ patients at early stages have achieved higher disease-free survival rate. Because of the complex anatomy of the head and neck, most tumor targets are very irregular, and IMRT has been shown to improve the quality of life for patients undergoing therapy by sparing the critical organs surrounding the tumor. Therefore, head and neck cancer has become the biggest beneficiary of this new technology. ${ }^{2-4}$ At present, IMRT technology is widely used in the treatment of head 
and neck tumors, and studies have confirmed that IMRT can improve the target dose and achieve an ideal therapeutic effect. $^{5-8}$ But treatment plans for head and neck cancer are highly complex due to multiple dose prescription levels and numerous OARs close to the target; ${ }^{9}$ each OAR has its own limiting dose, so the quality of treatment plan depends greatly on the skill and experience of clinical physicists, and clinical physicists need to spend plenty of time in order to make a high-quality treatment plan. As a result, IMRT planning can be a time-consuming and frustrating task, and the quality of treatment plans with similar target dose prescriptions and normal tissue constraints will vary greatly between physicists and institutions. ${ }^{10}$ The automated system's treatment plan can effectively reduce manual time and also reduce the difference between treatment plans designed by physicists through trial-and-error. It has been shown that it is possible to almost fully automate or accelerate this functionality where plans are created much faster and with greater consistency and quality. ${ }^{11}$ The research of auto-IMRT plans can be divided into two parts: one is to develop system algorithms to automatically generate and optimize model parameters, and the other is using the target parameters required for the new program which were extracted from a large number of treated patient planning database to optimize for new treatment plan. This research used mdaccAutoPlan system as a plug-in to the Pinnacle $^{3}$ treatment planning system (TPS), this system uses system algorithms and an iterative approach to optimize model parameters. It can set beam angle, planning structures, and objective function parameters automatically without human intervention. In terms of tumor coverage and normal tissue sparing, the computer-generated plans are intended to be better than, or at least equivalent to, those designed by physicists through trial-and-error. It is also our goal that the methodology is executable rapidly enough to be used in routine. Currently, automatic planning has been used in cervical cancer, ${ }^{12}$ and radiofrequency ablation planning of liver cancer ${ }^{13}$ and prostate cancer, ${ }^{14}$ and studies have shown that automatic planning achieves better results than manual planning. In this paper, we used the mdaccAutoPlan system to generate 105 IMRT autoplans of head and neck cancer, and then compared the dose distribution with IMRT manual plans, to explore the advantages of IMRT autoplans for head and neck cancer.

\section{Materials and Methods}

\section{Patient Characteristics}

From August 2015 to August 2018, 105 patients treated for nasopharyngeal carcinoma in our hospital were selected; the patients and their characteristics are reported in Table 1.

All procedures performed in studies involving human participants were in accordance with the ethical standards of the institutional and/or national research committee and with the 1964 Helsinki declaration and its later amendments or comparable ethical standards. The study was approved by the ethical committees of Sichuan Cancer Hospital and all patients provided written informed consent, in accordance with the Declaration of Helsinki and our editorial ethics policy.

\section{Planning Target Volume (PTV) Contouring and Prescription}

All PTV was contoured by experienced physicians in our hospital in accordance with "The common view and guideline of expert on target area and dose design for intensitymodulated radiation therapy for nasopharyngeal carcinoma 2010". 15 Planning gross tumor volume (PGTV), PGTVn was constructed from GTV, GTVn (bilateral neck nodes gross target volume) with the addition of a $3 \mathrm{~mm}$ margin, with the description dose from 68 Gy to 70 Gy, PCTV66 was constructed from clinical target volume (CTV) with the addition of a $3 \mathrm{~mm}$ margin, with the description dose of

Table I Patient Characteristics

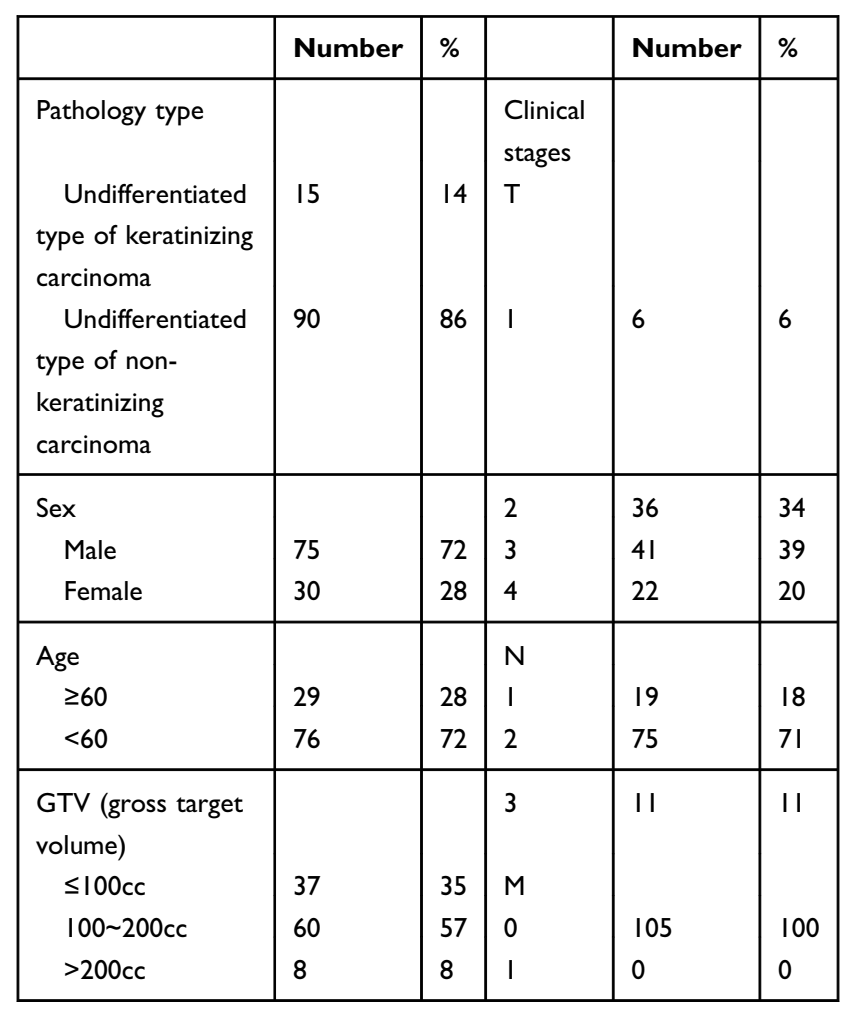


66 Gy, for PCTV56 and PCTVn, the description dose was $56 \mathrm{~Gy}$. The goal of our center is to reach at least $95 \%$ of the prescription dose of every PTV.

\section{OARs Dose Limiting}

The recommended plan assessment requirements for OAR followed in our center are shown in Table 2.

\section{Plan Design \\ Manual Plan Design}

For this study, 105 nasopharyngeal carcinoma treatment plans were selected, and all these cases were generated on the Eclipse treatment planning system (version A11.0). The treatment plans of all patients were generated by experienced medical physicists in our center and all the manual plans were optimized according to our standard clinical practice. All the basic parameters and beam angles of 105 patients were the same, the seven treatment beams were oriented at the angles of $153^{\circ}$, $102^{\circ}, 51^{\circ}, 0^{\circ}, 309^{\circ}, 258^{\circ}, 207^{\circ}$, and all beams were delivered at $6 \mathrm{MV}$ according to our center's standard.

\section{Auto Plan Design}

MdaccAutoPlan software provides a template with configurable parameters. Regions of interest (ROIs) (e.g. PTV or expanded cord), placement of points of interest (POIs), prescriptions, beam geometries, optimization options, and prioritized optimization goals can be defined automatically; the only inputs to the system were the delineations of planning target and organs at risk.

All of the 105 treatment plans were replanned by the mdaccAutoPlan system available in Pinnacle3 (version

Table 2 Dose Limit of OARs

\begin{tabular}{|l|l|l|}
\hline OAR & Max Dose & Limiting Dose \\
\hline Brain stem & 54 Gy & $>60 \mathrm{~Gy} \leq 1 \%$ \\
Spinal cord & $45 \mathrm{~Gy}$ & $>50 \mathrm{~Gy} \leq 1 \%$ \\
Optic nerve & $50 \mathrm{~Gy}$ & $55 \mathrm{~Gy}$ \\
Optic chiasm & $50 \mathrm{~Gy}$ & $55 \mathrm{~Gy}$ \\
Temporal lobe & $60 \mathrm{~Gy}$ & $>65 \mathrm{~Gy} \leq 1 \%$ \\
Lens & $25 \mathrm{~Gy}$ & \\
\hline & Mean dose & \\
\hline Pituitary & $\leq 50$ Gy & \\
\hline Parotid gland & Double sides<25 Gy when PTV was complicated \\
& (PTV overlap with parotid) as low as possible \\
\hline $\begin{array}{l}\text { Oral cavity } \\
\text { Cochlea }\end{array}$ & $\leq 40$ Gy & \multicolumn{2}{|l}{} \\
\hline
\end{tabular}

9.10). In order to reduce the influence on the final dose distribution, in this study, we used the same position of isocenter and same beam angles as the manual plan.

\section{Data Statistics}

All AU and MA plans were delivered to Matlab software. $\mathrm{D}_{2}$, $\mathrm{D}_{50}, \mathrm{D}_{95}, \mathrm{D}_{98}$ of PGTV, PCTV60, PCTV56, and PCTVn were calculated in Matlab, and their homogeneity index (HI), $\mathrm{HI}=\left(\mathrm{D}_{2}-\mathrm{D}_{95}\right) / \mathrm{D}_{50}$ and conformal index $(\mathrm{CI})^{17}$ of PGTV and PCTV56+PCTVn were calculated, where $\mathrm{CI}=$ $\left(\mathrm{V}_{\text {ptv }} / \mathrm{V}_{\text {ptv,ref }}\right) \times\left(\mathrm{V}_{\text {ref }} / \mathrm{V}_{\text {ptv,ref }}\right)$, Vptv, ref is the volume of the target area wrapped around the reference isodose line, Vptv is the target volume, and Vref is the total volume wrapped around the reference isodose line. The reference dose in this paper is the prescribed dose, so the higher the CI value, the worse the prescription dose conformal. We chose OARs of the brain stem, spinal cord, lens, temporal lobe, optic nerve, and optic chiasm to calculate their Dmax, and oropharynx, parotid, mandible joint, pituitary, thyroid, and cochlea to calculate their Dmean. Then, we recorded the monitor unit (MU) of the auto plan and the manual plan of every patient, and performed the $t$ test of the parameters above, where $P<0.05$ means the difference was significant.

\section{Results}

\section{Target Dose Comparison}

In terms of target coverage, $\mathrm{CI}, \mathrm{HI}$, and $\mathrm{MU}$ of 105 patients, there is no significant difference between $\mathrm{AU}$ and MA plans; the $\mathrm{D}_{95}, \mathrm{CI}$, and $\mathrm{HI}$ of AU and MA plans are shown in Tables 3 and 4 respectively. The monitor units of $\mathrm{MA}$ and $\mathrm{AU}$ plans are $1754.6 \pm 117.2$ and $1774.4 \pm 87.8$, respectively; $P=0.074$.

\section{OAR Dose Comparison}

Organs are divided into serial organs (brain stem, spinal cord, crystal, optic nerve, etc.) and parallel organs (parotid gland, oropharynx, mandible joint, etc.) in accordance with their radiation biological characteristics. The average value of serial organs maximum doses in 105 patients are shown in Table 5, and the average numbers of parallel organ doses are shown in Table 6. From Tables 5 and 6, we can see that all the

Table 3 Comparison of D95 of MA and AU Plan (X $\pm \chi)$

\begin{tabular}{|l|l|l|l|}
\hline Target & MA D95 (cGy) & AU D $_{95}$ (cGy) & $P$ \\
\hline PGTV & $6722.4 \pm 223.8$ & $6823.4 \pm 188.7$ & 0.2 \\
PGTVn & $6834.4 \pm 253.7$ & $6756.2 \pm 208.4$ & 0.3 \\
PCTV60 & $6067.4 \pm 464.4$ & $5992.2 \pm 452.2$ & 0.3 \\
PCTV56+PCTVn & $5623.7 \pm 593.4$ & $5605.6 \pm 528.1$ & 0.3 \\
\hline
\end{tabular}


Table 4 Comparison of $\mathrm{Cl}$ and $\mathrm{HI}$ for $\mathrm{AU}$ and MA Plan $(\mathrm{X} \pm \chi)$

\begin{tabular}{|l|l|l|l|}
\hline HI & MA Plan & AU Plan & $P$ \\
\hline PGTV+PGTVn & $0.0684 \pm 0.005$ & $0.0832 \pm 0.007$ & 0.11 \\
PCTV60 & $0.0986 \pm 0.006$ & $0.1542 \pm 0.006$ & 0.86 \\
PCTV56 & $0.1838 \pm 0.015$ & $0.1856 \pm 0.018$ & 0.69 \\
PCTVn & $0.2296 \pm 0.021$ & $0.2688 \pm 0.024$ & 0.06 \\
Cl & & & \\
PGTV+PGTVn & $1.35 \pm 0.21$ & $1.34 \pm 0.15$ & 0.92 \\
PCTVn & $1.54 \pm 0.27$ & $1.57 \pm 0.28$ & 0.14 \\
\hline
\end{tabular}

Table 5 Comparison of Max Dose for Serial Organs (cGy, X $\pm \chi$ )

\begin{tabular}{|l|l|l|l|}
\hline OARs & MA Plan & AU Plan & $P$ \\
\hline Brain stem & $4922.3 \pm 452.7$ & $4868.1 \pm 342.6$ & 0.7 \\
Spinal cord* & $3870.1 \pm 297.3$ & $2506.3 \pm 734.4$ & 0.0 \\
Left lens* & $478.3 \pm 159.0$ & $409.9 \pm 90.0$ & 0.0 \\
Right lens* & $472.5 \pm 164.1$ & $423.3 \pm 109.7$ & 0.0 \\
Left temporal lobe & $6328.0 \pm 450.3$ & $6327.0 \pm 429.8$ & 0.7 \\
Right temporal lobe & $6458.8 \pm 431.4$ & $6422.8 \pm 431.4$ & 0.3 \\
Left optic nerve* & $2412.3 \pm 1661.7$ & $2100.0 \pm 1503.1$ & 0.0 \\
Right optic nerve* & $2578.6 \pm 1865.4$ & $2071.1 \pm 1667.7$ & 0.002 \\
Optic chiasm & $2389.3 \pm 1692.2$ & $2331.3 \pm 1696.2$ & 0.42 \\
\hline
\end{tabular}

Note: *Indicates a statistically significant difference in maximum dose $(P<0.05)$.

Table 6 Comparison of Mean Dose for Parallel Organs (cGy, X \pm $\chi)$

\begin{tabular}{|l|l|l|l|}
\hline OARs & MA Plan & AU Plan & $P$ \\
\hline Oropharynx & $3328.9 \pm 313.4$ & $3126.5 \pm 313.0$ & $P=0.001$ \\
Left parotid gland* & $2859.2 \pm 313.2$ & $2322.3 \pm 506.0$ & $P=0.0$ \\
Right parotid gland* & $2845.6 \pm 334.6$ & $2452.5 \pm 431.9$ & $P=0.0$ \\
Left mandible* & $3913.4 \pm 432.2$ & $3105.0 \pm 470.4$ & $P=0.0$ \\
Right mandible* & $3884.0 \pm 401.9$ & $3087.6 \pm 407.2$ & $P=0.0$ \\
Left mandible joint* & $3661.1 \pm 631.4$ & $1794.6 \pm 709.4$ & $P=0.0$ \\
Right mandible joint* & $3631.5 \pm 709.2$ & $1767.4 \pm 824.3$ & $P=0.0$ \\
Pituitary & $2818.7 \pm 1283.2$ & $2643.9 \pm 1500.9$ & $P=0.158$ \\
Thyroid* & $2 \mid 43.5 \pm 561.9$ & $1667.5 \pm 332.7$ & $P=0.0$ \\
Left cochlea* & $3953.2 \pm 468.9$ & $3468.3 \pm 807.1$ & $P=0.0$ \\
Right cochlea* & $4032.3 \pm 493.7$ & $3513.5 \pm 752.6$ & $P=0.0$ \\
Normal tissue* (NT) & $932.1 \pm \mid 10.2$ & $814.1 \pm 79.9$ & $P=0.025$ \\
\hline
\end{tabular}

Note: *Indicates a statistically significant difference in mean dose $(P<0.05)$.

doses of OARs in AU plans are significantly lower than in MA plans, except for brain stem, optic chiasm, temporal lobe, and pituitary.

\section{Comparison of Dose-Volume Histogram (DVH)}

Below are three average DVHs of the parotid gland, mandible joint and spinal cord in the 105 planned cases
(Figure 1) which are representative of the comparison of dose distribution obtained in AU and MA plans.

\section{Comparison of Dose Distribution}

Figure 2 shows a comparison of isodose lines from a typical patient of AU and MA plans which intercepts a representative level of the same layer. It can be seen that the AU plan was visually more conforming and had better OAR sparing and steeper dose fall away from the targets.

\section{Comparison of MA Plans in Pinnacle TPS and Eclipse TPS}

In our research, AU plans were generated in Pinnacle TPS and MA plans were generated in Eclipse TPS, in order to observe if there is a significant difference between two systems in planning optimization; 20 patients were randomly selected from the 105 cases to do MA plans in Pinnacle TPS. The dose comparisons are as given in Tables 7-9.

\section{Discussion}

This study showed that there is no significant difference in target dose between AU and MA plans, but the AU plan has clear advantages in protecting OARs as well as saving labor time, as also concluded by Ilma Xhaferllari et al, ${ }^{18}$ Hazell et $\mathrm{al}^{19}$ and $\mathrm{He}$ et al. ${ }^{20}$ From the comparison of AU and MA plans, we can see that the AU plan has an important protective effect on the brain stem, spinal cord, temporal lobe, parotid gland, and other organs. For example, it can theoretically reduce radiation damage for the brainstem and spinal cord, the incidence of cognitive impairment may be reduced and it reduces the incidence of xerostomia and the chance of oral infection.

In this study, all patients of the AU plan were independently implemented with the mdaccAutoPlan automated planning software and met clinical requirements without any human intervention, which greatly reduces the working time. In the majority of radiotherapy institutions in China, the quality of radiotherapy planning is limited because of the large number of patients and limited planning time of the clinical physicists, but the automatic planning system not only can make it easier for radiotherapy institutions to make quality plans in limited resources, but also can give physicists more time to focus on quality assurance, rather than spending time in program optimization. If there are other clinical requirements, it can be directly adjusted and optimized further on the original plan. Therefore, AU planning can be treated as 


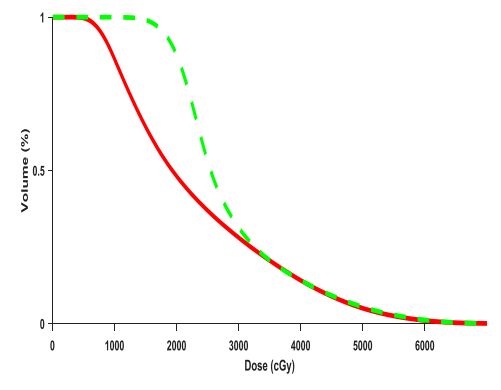

A

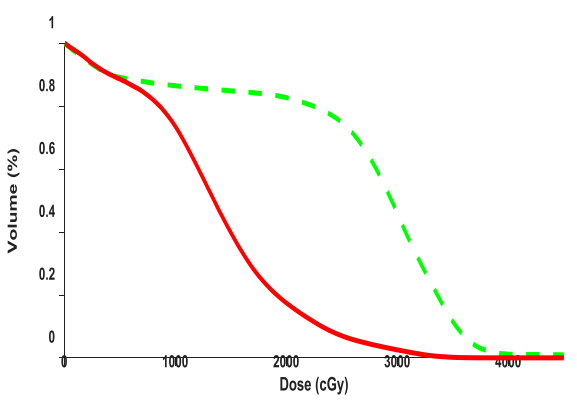

B

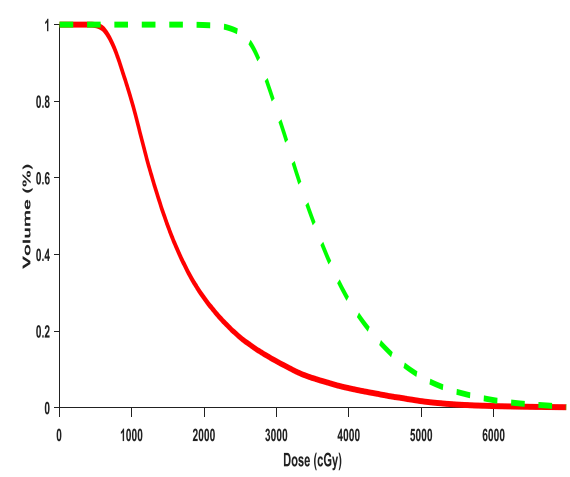

C

Figure I (A) Comparison of parotid gland DVH showing that the parotid gland received a higher dose in MA plans. (B) Comparison of spinal cord DVH showing that spinal cord received a higher dose in MA plans. (C) Comparison of mandible joint DVH showing that mandible joint received a higher dose in MA plans. The red line represents the $\mathrm{AU}$ plan and the green line represents the MA plan.

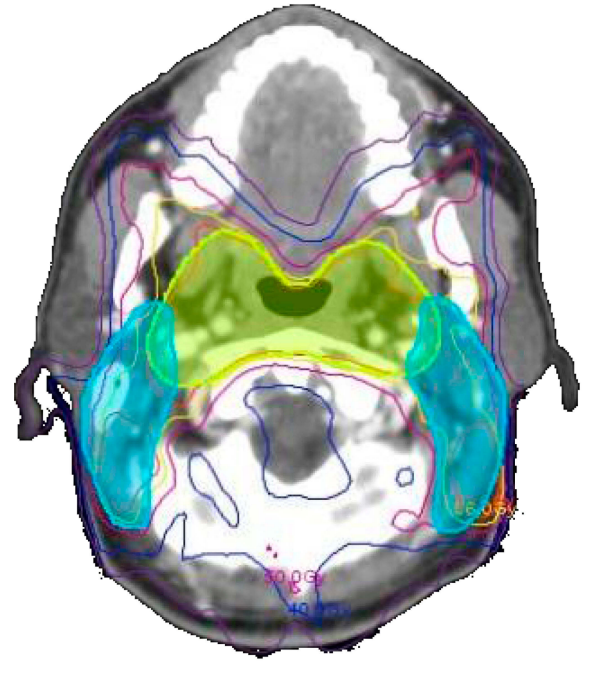

A

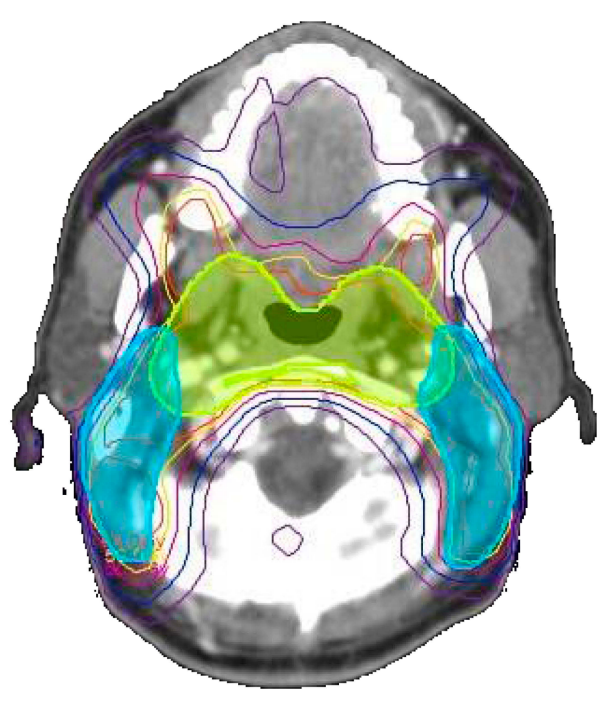

B

Figure 2 (A) Dose distribution of MA plans (B) Dose distribution of AU plans $\square$ PCTVI,

PCTV2.

a high-quality treatment plan that meets clinical needs without any human intervention and can also be used as a starting point for further manual adjustment. ${ }^{19}$ In addition, Zhu Jian et $\mathrm{al}^{22}$ studied the ART plan dose evaluation method based on the script function in the Pinnacle planning system, and concluded that the ART plan evaluation method is accurate, simple, and convenient, and improves the flexibility and accuracy of evaluating the dose of adaptive radiotherapy; it can also provide the technical support for clinical and scientific research work.

Manual adjustment of nasopharyngeal carcinoma requires the high-level experience of physicists. As shown in Figure 1, the parotid received a lower dose in a low-dose area, which shows that for low-dose areas, there is still some room to improve after manual optimization. The spinal cord received a lower mean dose because the physicist focused on the maximum dose in the spinal cord when manually optimizing. When beginning optimization, physicists need to ensure the target coverage; however, due to limited time the organ limit is relatively loose, and physicists cannot guarantee that the lowdose limit is maintained in every organ. This may result in some low tolerance dose OARs (like mandible joint) receiving higher doses. However, all of the 105 manual plans have met the clinical requirements, but still can be improved. A study on IMRT breast cancer ${ }^{21}$ showed that an automatic planning system provided great help to less experienced 
Table $7 \mathrm{Cl}$ and $\mathrm{HI}$ for MA Plans in Eclipse and Pinnacle TPS

\begin{tabular}{|l|l|l|l|}
\hline HI & Eclipse & Pinnacle & $P$ \\
\hline PGTV & $0.0777 \pm 0.006$ & $0.0858 \pm 0.006$ & $P=0.24$ \\
PCTVI & $0.0704 \pm 0.005$ & $0.0644 \pm 0.003$ & $P=0.13$ \\
PCTV2 & $0.1548 \pm 0.023$ & $0.1651 \pm 0.047$ & $P=0.62$ \\
PCTVn & $0.2361 \pm 0.030$ & $0.2672 \pm 0.032$ & $P=0.15$ \\
Cl & & & \\
PGTV & $1.36 \pm 0.11$ & $1.37 \pm 0.21$ & $P=0.46$ \\
PCTVn & $1.54 \pm 0.23$ & $1.52 \pm 0.25$ & $P=0.21$ \\
\hline
\end{tabular}

Table 8 Max Dose of Serial Organs for MA Plans in Eclipse and Pinnacle TPS cGy, $(X \pm \chi)$

\begin{tabular}{|l|l|l|l|}
\hline OARs & Eclipse & Pinnacle & $P$ \\
\hline Brain stem & $4933.3 \pm 452.7$ & $5056.9 \pm 239.8$ & $P=0.5$ \\
Spinal cord & $3854.1 \pm 297.3$ & $3875.5 \pm 537.3$ & $P=0.5$ \\
Left lens & $47 I .3 \pm 159.0$ & $456.7 \pm 35.5$ & $P=0.3$ \\
Right lens & $455.5 \pm 164.1$ & $427.3 \pm 64.4$ & $P=0.5$ \\
Left temporal lobe & $2428.3 \pm 1661.7$ & $2338.8 \pm 1285$ & $P=0.3$ \\
Right temporal lobe & $2564.6 \pm 1865.4$ & $25|2.8 \pm| 1454.5$ & $P=0.2$ \\
Left optic nerve & $2368.3 \pm 1692.2$ & $23|8.1 \pm| 140.3$ & $P=0.2$ \\
\hline
\end{tabular}

Table 9 Mean Dose of Parallel Organs Ofor MA Plans in Eclipse and Pinnacle TPS (cGy, X $\pm \chi$ )

\begin{tabular}{|l|l|l|l|}
\hline OARs & Eclipse & Pinnacle & $P$ \\
\hline Oropharynx & $3348.9 \pm 313.4$ & $3326.7 \pm 272.4$ & $P=0.8$ \\
Left parotid gland & $2847.12 \pm 313.2$ & $2913.8 \pm 467.3$ & $P=0.6$ \\
Right parotid gland & $2872.68 \pm 334.6$ & $2856.4 \pm 475.7$ & $P=0.3$ \\
Left mandible & $3926.43 \pm 432.2$ & $3826.8 \pm 922.2$ & $P=0.07$ \\
Right mandible & $3856.05 \pm 401.9$ & $3776.8 \pm 819.9$ & $P=0.08$ \\
Left mandible joint & $3637.1 \pm 631.4$ & $3724.6 \pm 854.4$ & $P=0.2$ \\
Right mandible joint & $3644.5 \pm 709.2$ & $3705.3 \pm 792.3$ & $P=0.1$ \\
Normal tissue (NT) & $946.1 \pm 110.2$ & $982.4 \pm 118.8$ & $P=0.6$ \\
\hline
\end{tabular}

physicists, but for physicists with rich experience, the results showed small differences between AU and MA plans. If there are enough time and experience for physicists to adjust treatment planning, physicists can generate treatment plans with the same high quality as automatic systems.

An AU plan can guarantee the premise of the target coverage as long as tightening all OARs' dose limit, it can also reduce the various dose distributions between the treatment plans in quality assurance during limited time, and this is the biggest advantage of AU planning. On the other hand, the survival period of head and neck cancer patients is relatively long; clinically, doctors have paid more attention to improving the quality of patients' life, and the results of this study can provide a quantitative reference to the clinical evaluation.
Bo Penggang ${ }^{23}$ and $\mathrm{He} \mathrm{Genbo}^{20}$ also studied automatic IMRT planning for nasopharyngeal carcinoma and got good results, the former is a combination of the $\mathrm{C}++$ language and the Pinnacle ${ }^{3}$ treatment plan system script, and the latter is programmed to run automatically. This study uses an automatic program system mdaccAutoPlan, without programming with a large number of patients treated plan collection to be the database, Bo Penggang et $\mathrm{al}^{23}$ and $\mathrm{He}$ et $\mathrm{al}^{20}$ has the collection of 10 cases, this study collected 105 cases of patients, which can reduce the random error of data.

In the choice of the automatic IMRT plan angle, unlike Zhang et al, ${ }^{24}$ in order to reduce the effect of AU planning and MA planning results due to the different field angle, this study did not use the angular optimization function in the mdaccAutoPlan automatic planning system, the NPC target distribution of AU plan and MA plan in this study were unified by 7 equipartition field, whether the optimization results with different beam number and angle will be better? This is our next step to continue the study.

Deficiency, the AU plan and the MA plan were calculated using two planning systems (Pinnacle and Eclipse, respectively), we have compared the dose of MA plans between Pinnacle and Eclipse TPS, as shown in Tables 79, there is no significant differences between these two systems, and research of Lopez et $\mathrm{al}^{25}$ also have shown that the difference in dose calculation between the two planning systems of Pinnacle and Eclipse is less than $3 \%$, the AU and MA plans are comparable. However, this study only compared the optimization results of the two systems, but not for the research on the algorithms and clinical use, which will be the direction of our further research. We have not yet performed a quality assurance of AU plans yet; we will also do further research to see if it can be used in clinical applications.

\section{Conclusion}

Comparison of AU and MA plan for nasopharyngeal cancer shows a very small dosimetric difference in target coverage, but the AU plan has clear advantages in protecting OARs as well as saving labor time. It may be another option for the clinical implementation of nasopharyngeal carcinoma IMRT treatment planning.

\section{Disclosure}

The abstract has been presented previously as an e-poster in ESTRO 2017. The authors report no conflicts of interest in the work. 


\section{References}

1. Lee N, Harris J, Garden AS, et al. Intensity-modulated radiation therapy with or without chemotherapy for nasopharyngeal carcinoma: radiation therapy oncology group Phase $\square$ trail. $J$ Clin Oncol. 2009;27:3684-3690. doi:10.1200/JCO.2008.19.9109

2. Zhiyong Y, Li G, Guozhen X, et al. Preliminary results of intensity modulated radiation therapy for untreated nasopharyngeal carcinoma. Chin J Oncol. 2008;15(14):237-238.

3. Lin A, Kim HM, Terrell JE, et al. Quality of life after parotid-sparing IMRT for head-and-neck cancer: a prospective longitudinal study. Int $J$ of Rad Onc Biol Phys. 2003;57(1):61-70. doi:10.1016/S0360-3016(03) 00361-4

4. Parliament M, Scrimger RA, Anderson SG, et al. Preservation of oral health-related quality of life and salivary flow rates after inverseplanned intensity-modulated radiotherapy (IMRT) for head-and-neck cancer. Int J Rad Onc Biol Phys. 2004;58(3):663-673. doi:10.1016/ S0360-3016(03)01571-2

5. Shaojun L, Chuaben C, Han L, et al. Preliminary results of intensity modulated radiotherapy for nasopharyngeal carcinoma: a report of 230 cases. J Fujian Med Univ. 2007;41(1):66-70.

6. Lee N, Xia P, Fischbein NJ, et al. Intensity modulated radiation therapy for head-and-neck cancer: the UCSF experience focusing on target volume delineation. Int $J$ of Rad Onc Biol Phys. 2003;57 (1):158-168. doi:10.1016/S0360-3016(03)00405-X

7. Huang D, Xia P, Akazawa P, et al. Comparison of treatment plans using intensity-modulated radiotherapy and three-dimensional conformal radiotherapy for para nasal sinus carcinoma. Int J Radiat Oncol Biol Phys. 2003;56(1):158-168. doi:10.1016/S0360-3016(03)00080-4

8. Chong Z, Taiiang L, Fei H, et al. Clinical study of 139 nasopharyngeal carcinoma patients for intensity modulated radiation therapy. Chin J Oncol. 2006;15(1):1-6.

9. Hansen CR, Bertelsen A, Hazell I, et al. Automatic treatment planning improves the clinical quality of head and neck cancer treatment plans. Clin Transl Radiat Oncol. 2016;1:2-8. doi:10.1016/j.ctro.2016.08.001

10. Schwarz M 2009 Clinical implementation: how can IMRT become routine? medicalphysicsweb http://medicalphysicsweb.org/cws/arti cle/opinion/37799.

11. Xhaferllari L, Wong E, Bzdusek K, Lock M, Chen JZ. Automated IMRT planning with regional optimization using planning scripts. Appl Clin Med Phys. 2013;14(1):331-346.

12. Wu B, Ricchetti F, Sanguineti G, et al. Patient geometry-driven information retrieval for IMRT treatment plan quality control. Med Phys. 2009;36(12):5497-5505. doi:10.1118/1.3253464

13. Petit SF, Wu B, Kazhdan M, et al. Increased organ sparing using shape-based treatment plan optimization for intensity modulated radiation therapy of pancreatic adenocarcinoma. Radiother Oncol. $2012 ; 102: 38-44$
14. Wu B, Ricchetti F, Sanguineti G, et al. Data-driven approach to generating achievable dose-volume histogram objectives in intensity-modulated radiotherapy planning. Int J Radiat Oncol Biol Phys. 2011;79:1241-1247. doi:10.1016/j.ijrobp.2010.05.026

15. Clinical staging Committee of nasopharyngeal carcinoma in China. Expert consensus on target area and dose design guideline of intensity modulated radiotherapy for nasopharyngeal carcinoma 2010 . Chin J Oncol. 2011;20(4):267-269.

16. Radiation Therapy Oncology Group. A Phase II studies of concurrent chemo radiotherapy using three. Dimensional conformal radiotherapy (3D-CRT) or intensity modulated radiation. Therapy (IMRT) +Bevaciztanab(BV)for locally or regionally advanced nasopharyngeal cancer. RTOG. Available from: http://208.251.169.72 $\square \mathrm{mem}$ bers $\square$ pmtoeols $\square 0615 \square 0615$.

17. Zengjun Z, Jianbo W, Yufeng C, et al. Osimetric comparison and analysis of two kinds of intensity-modulated plan for nasopharyngeal carcinoma. Chin J Radiol Health. 2014;12(23):525-527.

18. Xhaferllari I, Wong E, Bzdusek K, et al. Automated IMRT planning with regional optimization using planning scripts. Appl Clin Med Phys. 2013;14(1):176-191. doi:10.1120/jacmp.v14i1.4052

19. Hazell I, Bzdusek K, Kumar P, et al. Automatic planning of head and neck treatment plans. Appl Clin Med Phys. 2016;17(1):272-282. doi:10.1120/jacmp.v17i1.5901

20. He Y, Longbin Z, Jianghong X, et al. Feasibility of automatic treatment planning in intensity-modulated radiotherapy of nasopharyngeal carcinoma. J Biomed Eng. 2015;32(6):1288-1293.

21. Wang J, Hu W, Yang Z, et al. Is it possible for knowledge-based planning to improve intensity modulated radiation therapy plan quality for planners with different planning experiences in left-sided breast cancer patients? Radiat Oncol. 2017;12(1):85. doi:10.1186/ s13014-017-0822-Z

22. Jian Z, Tong B, Zhen H, et al. Dose evaluation method for adaptive radiotherapy plans based on script functions. Chin J Oncol. 2016;25 (9):984-988

23. Bo Penggang. Programming of automatic planning basing on datadriven for intensity-modulated radiotherapy for nasopharyngeal carcinoma. Med Equip. 2013;26(2):6-9.

24. Zhang X, Li X, Quan EM, et al. A methodology for automatic intensity-modulated radiation treatment planning for lung cancer. Phys Med Biol. 2011;56(13):3873-3893. doi:10.1088/0031-9155/56/ $13 / 009$

25. Lopez PM. Dosimetric Comparison of Pinnacle 3 9.2, Eclipse TM 11.0 And iplan 4.1 Algorithms with Heterogeneous Phantoms. 2014.

\section{Publish your work in this journal}

Cancer Management and Research is an international, peer-reviewed open access journal focusing on cancer research and the optimal use of preventative and integrated treatment interventions to achieve improved outcomes, enhanced survival and quality of life for the cancer patient.
The manuscript management system is completely online and includes a very quick and fair peer-review system, which is all easy to use Visit http://www.dovepress.com/testimonials.php to read real quotes from published authors. 